Abstract 121 Table 1

\begin{tabular}{llll}
\hline Variables & $\begin{array}{l}\text { Before } \mathbf{N}=\mathbf{4 4} \\
\text { long-stay patients }\end{array}$ & $\begin{array}{l}\text { After } \mathbf{N}=\mathbf{4 8} \\
\text { long-stay patients }\end{array}$ & p-value \\
\hline Patient age in months, median (IOR) & $6(0$ to 99$)$ & $2(0$ to 48$)$ & 0.40 \\
Girls, $n$ (\%) & $21(47.7 \%)$ & $22(45.8 \%)$ & 0.86 \\
Length of stay, median (IOR) & $26(20$ to 42$)$ & $22(15$ to 33$)$ & 0.036 \\
Primary care nurse assigned, n (\%) & $25(56.8 \%)$ & $16(33.3 \%)$ & 0.024 \\
Start day primary care, median (IQR) & $12(8$ to 16$)$ & $12(8$ to 18$)$ & 0.76 \\
\hline
\end{tabular}

Conclusions Regrettably, the awareness week did not bring about improvement in compliance with assigning a primary care nurse. On the contrary, the compliance was worse. Therefore we need to consider other strategies in the assignment procedure, which is now on a voluntary basis.

\section{THE RIGHTS OF HOSPITALIZED CHILDREN. A SURVEY ON THEIR IMPLEMENTATION IN ITALIAN PEDIATRICS UNITS}

doi:10.1136/archdischild-2012-302724.0122

${ }^{1} \mathrm{~F}$ Festini, ${ }^{2} \mathrm{C}$ Aringhieri, ${ }^{2} \mathrm{~S}$ Bisogni. IItalian Society of Pediatric Nursing Science - SISIP, Pistoia; '2University of Florence, Florence, Italy

Background Several "Charts of rights" have been issued in Europe to solemnely proclaim the Rights of children during their hospital stay. However, notwithstanding such general declarations, the actual implementation of hospitalized children's rights is unclear. Nurses play a fundamental role in putting into effect the Charts of rights of hospitalized children.

Goal. To evaluate to what extent the rights of hospitalized children are actually respected in Italian Pediatrics Units.

Methods Cross-sectional study. The study was promoted by Italian Society of Pediatric Nursing Science (SISIP). A 12-item online questionnaire was set up and an invitation was sent by email to pediatric Nurses using SISIP's mailing lists. Responders indicated to what extent each right is respected in their hospital using a numeric scale from 1 (never) to 5 (always).

Results 536 questionnaires were returned. The best implemented right is the right of children to have their mothers with them (mean score 4.47). The least respected is the right of children to express their opinion about care (mean 3.01). Other rights considered: right to play (4.29), right of information (3.95), right to the respect of privacy (3.75), right to be hospitalized with peers (3.4), right not to experience pain ever (3.24), right to school (3.08). According to the majority of Nurses, the most important is the right to pain relief.

Discussion Despite official declarations, the rights of hospitalized children are far from being enforced in Italian Pediatrics Unit. Nurses must proactively participate to the respect of such rights, in particular the right to pain relief.

\section{WEANING FROM VENTILATION: A DEVELOPING ROLE FOR PEDIATRIC INTENSIVE CARE UNIT (PICU) NURSES? EVIDENCE FROM TWO COCHRANE REVIEWS}

doi:10.1136/archdischild-2012-302724.0123

B Blackwood. School of Nursing and Midwifery, Queen's University, Belfast, UK

Background Mechanical ventilation (MV) carries potential risks to mortality and morbidity; therefore, weaning should not be delayed. To safely reduce ventilator support, practice has transitioned from individual preference to a structured approach with guidelines.

Objectives To highlight international challenges in developing PICU nurses' role in weaning children from MV by reviewing the prevalence of, and evidence for, weaning protocols, and the current state of nurses' roles and responsibilities in ventilator weaning.

Main body Protocolised weaning has shown some success in reducing MV duration in adults and children. Consequently protocols have gained popularity with surveys reporting their use in $56-69 \%$ of European adults ICUs and 18\% of UK PICUs. Findings from two systematic reviews show support for weaning protocols in adults, but that cannot yet be said regarding children. There are only a small number of randomised trials of protocolised weaning in children; they used diverse protocols and reported discordant findings making it impossible to pool results. Internationally, there is insufficient information about PICU nurses' role in weaning, but a recent UK survey reported that nurses rarely titrated ventilator settings. It is possible that reticence to actively engage in the weaning process is linked to associated risks with pediatric extubation, but does not explain why nurses cannot progress weaning to the point of extubation.

Key challenges If paediatric nurses are to confidently engage in the process of weaning they require suitable training and support. Developing appropriate protocols may be an important vehicle for safely changing practice in this respect.

\section{EARLY INFLUENCES ON ASTHMA}

doi:10.1136/archdischild-2012-302724.0124

S Turner. University of Aberdeen, Aberdeen, UK

Childhood asthma is a common condition where symptoms are often present from preschool years and continue into adult life for many individuals. Asthma can be treated but not cured and the most promising means to reduce asthma prevalence is prevention. This talk will address two key questions relevant to asthma prevention: "what are the early influences on asthma?" and "when are they acting?". The focus will be on the fetal and preschool years and will include interactions between genetic and environmental factors. The audience will gain an understanding of the complexity of the early origins of asthma and also take home some (hopefully useful) practical advice for parents and governments.

\section{COPD IN THE NEXT 50 YEARS-SHOULD WE BLAME THE NEONATOLOGISTS?}

doi:10.1136/archdischild-2012-302724.0125

EJLE Vrijlandt. Department of Pulmonology, University Medical Center Groningen, Groningen, The Netherlands

In this talk, the current knowledge of respiratory sequelae following preterm birth in adulthood will be summarized. Specifically it will review respiratory symptoms, pulmonary function, exercise capacity and structural lung disease as determined by high resolution computed tomography.

How much of the problems of ex-preterms are due to natural causes, how much to iatrogenic causes? Of these two items, it is the natural influences that are studied most. A large number of cohort studies showed several themes that may have impact on lung development: antenatal factors such as the effects of smoking on airway anatomy and the fetal immune system, gene-environment interactions and post natal exposures. In this talk however, the focus will be on an area of growing interest- the iatrogenic long term influences on lung health. I mention here the follow up of neonatal intensive care but others exist such as the long term effects of lung transplantation.

During the talk data will be demonstrated showing that

- ex-preterms do have more respiratory symptoms, also later in life and that the preterms with the lowest mean birth weight do have the most symptoms. 
- a significantly greater proportion of children with BPD compared with those preterm children without BPD had a clinically important reduction in their mean percentage predicted FEV1

- Exercise capacity is not completely normal, but the differences are more subtle than expected.

- Ct scans show linear and triangular opacities, gas trapping and mosaic perfusion, emphysema especially in severe BPD, which represent subjects with old and new BPD.

Perhaps the most important question for clinicians in the audience is "can we do anything to prevent long term sequelae and if not what should I do to explore the long term sequelae in the most efficient way?" At the end of the talk, it will be clear that preventive possibilities are scarce. However in order to improve long-term respiratory outcome and health-related quality of life of all preterm infants, efforts should be aimed at

- preventing harmful viral lower respiratory infections during the first year of life

- Reducing cigarette smoke exposure

- Parental education that may increase awareness of disease

The EFCNI White Paper on Maternal and Newborn Health and Aftercare Services states that a worldwide network of long-term follow-up research into preterm children is needed. Long-term follow-up research into societal participation of adolescents born preterm should take place. The results can be used to develop interventions for teenagers born preterm to support them in their course of life towards adulthood; for example, by assigning job or life coaches. Research of long-term medical and social treatment of preterm children is needed and also guidelines for treatment and counseling by neonatologists, paediatricians, general physicians, well baby clinics and teachers should be developed.

\section{RANDOMIZED CONTROLLED TRIAL OF DAY-CARE AND HOSPITALIZED MANAGEMENT OF SEVERE PNEUMONIA WITH SEVERE ACUTE MALNUTRITION IN CHILDREN IN BANGLADESH}

doi:10.1136/archdischild-2012-302724.0126

${ }^{1} \mathrm{H}$ Ashraf, ${ }^{1} \mathrm{NH}$ Alam, ${ }^{2} \mathrm{~N}$ Gyr. ${ }^{1}$ Centre for Nutrition and Food Security (CNFS), International Centre for Diarrhoeal Disease Research, Bangladesh (icddr, b), Dhaka, Bangladesh; 'Department of Internal Medicine, University of Basel, Basel, Switzerland

Background and aims Severe childhood pneumonia and severe acute malnutrition (SAM) require hospitalized management but inadequate pediatric beds limits hospital-care in Bangladesh. As two prospective observational studies showed that day-care facility-based management of severe pneumonia and SAM were successful as alternatives to hospitalization, a RCT was conducted.

Methods A randomized hospital (ICHSH) versus day-care (The Radda Clinic equipped with oxygen, suction, pulse oximeter, nebulizer, glucometer) comparative study was carried out to evaluate the safety and effectiveness of day-care model. Children aged 2-59 months having severe pneumonia with SAM were randomized to day-care or hospital-care. Parents brought children at 08:00 at daycare clinic and back home at 17:00 daily after receiving antibiotics, diet, micronutrients and oxygen. For hospital-care, children received similar 24-hour treatment with antibiotics, diet, micronutrients and oxygen daily. Both day-care and hospital management continued daily until improvement and discharged.

Results In total, 340 children were randomized to either day-care or hospital-care management. Successful management was possible in 136/170 [80\% (95\% CI 73.4-85.3\%)] day-care children and 144/170 [84.7\% (95\% CI 78.5-89.3\%)] hospital-care children. Of remaining 34 day-care children, 29 [17.1\% (95\% CI 12.1-23.4\%)] were referred to hospital and 5 [2.9\% (95\% CI 1.3-6.7\%)] discontinued treatment. Of remaining 26 hospital-care children, 18 [10.6\%
(95\% CI $6.8-16.1 \%)]$ were referred to specialized hospitals and 6 [3.5\% (95\% CI 1.6-7.5\%)] discontinued treatment.

Conclusion Children with severe pneumonia with SAM could be treated safely and effectively on a day-care basis at established daycare clinics, similar to hospital management, if required logistic support is available.

\section{TRENDS IN SMOKING IN PREGNANCY IN THE NETHERLANDS (2001-2010)}

doi:10.1136/archdischild-2012-302724.0127

${ }^{1} \mathrm{Cl}$ Lanting, ${ }^{1} \mathrm{JP}$ van Wouwe, ${ }^{2}$ van den Burg, ${ }^{2} \mathrm{D}$ Segaar, ${ }^{1} \mathrm{KM}$ van der Pal-de Bruin ${ }^{1}$ Netherlands Institute for Applied Scientific Research TNO, Leiden; ${ }^{2}$ Stivoro, Den Haag, The Netherlands

Background and aims Smoking in pregnancy significantly increases the risk of preterm birth and fetal growth restriction. Pregnant women are encouraged to quit smoking. Smoking in general is discouraged by antismoking laws. In the present study, we describe trends in smoking in pregnancy in the Netherlands for 2001-2010. Methods National surveys in 2001, 2002, 2003, 2005, 2007, and 2010. In well baby clinics, questionnaires were handed out to mothers with infants $\leq 6$ months.

Results Out of a total number of 28,720 questionnaires, 16,358 (57\%) were returned. Between 2001 and 2010, prevalence of smoking in pregnancy dropped by half; from $13.0 \%$ in 2001 to $6.3 \%$ in 2010 $\left(\mathrm{P}_{\text {trend }}<0.001\right)$. The odds of being a smoker was 6.3 (95\%CI 5.3-7.4) for mothers with a low education level, and 3.0 (95\% CI 2.5-3.5) for mothers with a medium education level, as compared to mothers with high education level. Independently of their educational level, mothers smoked on average five cigarettes per day while pregnant.

We observed a sharp decrease in prevalence of smoking in pregnancy from 2003 to 2005 among women with a medium or a high education level. An almost continuous, downward trend was seen among mothers with a low level education. The 2003-2005 decrease coincided with the implementation of antismoking laws in the Netherlands.

Conclusions Between 2001 and 2010, prevalence of smoking in pregnancy dropped by half. But still, in 2010, 6.3\% of Dutch pregnant women were smokers, exposing about 11,000 unborn children per year to significantly increased health risks.

\section{INTRAPERITONEAL ADMINISTRATION OF CYTIDINE 5'-DIPHOSPHOCHOLINE (CDP-CHOLINE) REDUCES HYPEROXIC LUNG INJURY IN A NEONATAL RAT MODEL OF BRONCHOPULMONARY DYSPLASIA}

doi:10.1136/archdischild-2012-302724.0128

${ }^{1} \mathrm{M}$ Cetinkaya, ${ }^{2} \mathrm{M}$ Cansev, ${ }^{1} \mathrm{C}$ Tayman, ${ }^{1} \mathrm{~F}$ Cekmez, ' $\mathrm{FE}$ Canpolat, ${ }^{2} \mathrm{M}$ Kafa, ${ }^{3} \mathrm{~S}$ Uysal, 'SU Sarıcı. ' GATA Teaching Hospital, Ankara; ${ }^{2}$ Uludağ University Medical Faculty, Bursa; ${ }^{3}$ Fatih University Medical Faculty, Ankara, Turkey

Purpose The purpose of this study was to evaluate the preventive effect of CDP-choline treatment on hyperoxic lung injury, inflammation and apoptosis in a neonatal rat model of bronchopulmonary dysplasia BPD

Methods A total of 30 newborn pups were arranged in control, hyperoxia, and hyperoxia+CDP-choline groups. Immediately after birth, pups in the control group were kept in room air containing $21 \% \mathrm{O}_{2}$ and received daily saline injections, while those in hyperoxia and hyperoxia+CDP-choline groups were exposed to $95 \% \mathrm{O}_{2}$ and received daily injections of saline and CDP-choline $(300 \mathrm{mg} / \mathrm{kg})$, respectively, throughout postnatal day 10 (P10). Histopathological scoring, radial alveolar count, lamellar body protein expression, fibrosis, proinflammatory cytokine levels, oxidant/antioxidant enzyme activities, malondialdehyde content and apoptosis were evaluated on lung samples obtained at P10. 